\title{
Endoscopic balloon dilations for strictures of rectum, ileocecal valve and duodenum in a patient with X-linked inhibitor of apoptosis deficiency: a case report
}

\author{
Shinsuke Otagiri ${ }^{1}$, Takehiko Katsurada ${ }^{2}$, Kensuke Sakurai $^{1}$, Junichi Sugita ${ }^{3}$, Naoya Sakamoto ${ }^{1}$ \\ ${ }^{I}$ Department of Gastroenterology and Hepatology, Hokkaido University Graduate School of Medicine, Sapporo; Departments of \\ ${ }^{2}$ Gastroenterology and Hepatology and ${ }^{3}$ Hematology, Hokkaido University Hospital, Sapporo, Japan
}

\begin{abstract}
X-linked inhibitor of apoptosis (XIAP) deficiency is a rare primary immunodeficiency and gastrointestinal (GI) lesions in XIAP deficiency are similar to Crohn's disease. For patients with Crohn's disease, endoscopic balloon dilation (EBD) is known to be a standard procedure for intestinal strictures including upper GI tract. However, there are no articles which mention the efficacy of EBDs for the strictures in upper GI tract in patients with XIAP deficiency. Herein, we describe an 18-year-old male with XIAP deficiency in whom EBDs for the rectum, ileocecal valve (ICV), and duodenum were performed. Before hematopoietic stem cell transplantation (HSCT), GI endoscopy revealed strictures of the rectum, ICV and duodenum with active ulcers. Although these ulcers healed after HSCT, the strictures progressed. Therefore, we performed EBDs for the strictures of the rectum, ICV, and duodenum. In contrast studies, we did not find any other strictures in the small intestine. Throughout the patient's clinical course, no complications of EBD occurred. He started eating after EBDs, but abdominal symptoms did not relapse without any dietary restrictions. Our case suggests that EBD could be an effective and safe procedure for intestinal strictures including upper GI tract after HSCT in patients with XIAP deficiency. (Intest Res 2022;20:274-277)
\end{abstract}

Key Words: Endoscopy; Gastrointestinal diseases; Hematopoietic stem cell transplantation; X-linked inhibitor of apoptosis protein; Case reports

\section{INTRODUCTION}

X-linked inhibitor of apoptosis (XIAP) deficiency is a rare primary immunodeficiency, and the incidence of mutations in XIAP is estimated to be $1-2$ cases per million live births. ${ }^{1}$ The main clinical manifestations are hemophagocytic lymphohistiocytosis, splenomegaly, and gastrointestinal (GI) lesions. The clinical and histological features of GI lesions in patients with XIAP deficiency are similar to those in patients with Crohn's disease (CD). ${ }^{2}$ It was demonstrated that $4 \%$ of male patients

Received February 18, 2021. Revised March 9, 2021. Accepted April 14, 2021 Correspondence to Takehiko Katsurada, Department of Gastroenterology and Hepatology, Hokkaido University Hospital, N14, W5, Kita-ku, Sapporo 060-8638, Japan. Tel: +81-11-716-1161, Fax: +81-11-706-7867, E-mail:

tkatsu@amber.plala.or.jp with pediatric-onset $\mathrm{CD}$ had private variants in $\mathrm{XIAP}^{3}$ GI lesions in XIAP deficiency are generally severe and drug-resistant, and the only curative treatment is hematopoietic stem cell transplantation (HSCT). ${ }^{4}$

It is possible that patients with XIAP deficiency may develop intestinal strictures like those with $\mathrm{CD}^{5}{ }^{5}$ In patients with $\mathrm{CD}$, endoscopic balloon dilation (EBD) is known to be a standard procedure for intestinal strictures. ${ }^{6}$ We reported another case of a patient with XIAP deficiency who underwent EBDs after HSCT for a stricture of the ileocecal valve (ICV). ${ }^{7}$ However, there are no articles which mention the safety and efficacy of EBDs for the intestinal strictures including upper GI tract in patients with XIAP deficiency. Herein, we describe a case of XIAP deficiency in whom EBDs for multiple strictures of rectum, ICV and duodenum were performed after HSCT. 
The study's protocol was waived by the Institutional Review Board of Hokkaido University Hospital. Written informed consent was obtained.

\section{CASE REPORT}

An 18-year-old boy with XIAP deficiency was referred to our hospital for HSCT. He was diagnosed as having CD when he was 11 years of age in another hospital. He had been treated with various treatments such as steroids, azathioprine, infliximab, adalimumab, or ustekinumab. Nevertheless, clinical remission had never been achieved. Moreover, he repeatedly developed hemophagocytic lymphohistiocytosis. Therefore, genetic analysis was performed, and it revealed XIAP deficiency.

Before HSCT, colonoscopy revealed multiple active ulcers in the large intestine (Fig. 1A and B), a mild stricture of the rectum (Fig. 2A) and a severe stricture of the ICV (Fig. 3A) with active ulcers. Moreover, esophagogastroduodenoscopy revealed a mild stricture in the duodenum with an active ulcer (Fig. 4A). Due to these strictures and multiple ulcers, he could only consume a concentrated liquid diet. After HSCT, the strictures of the rectum (Fig. 2B) and duodenum (Fig. 4B) progressed, and
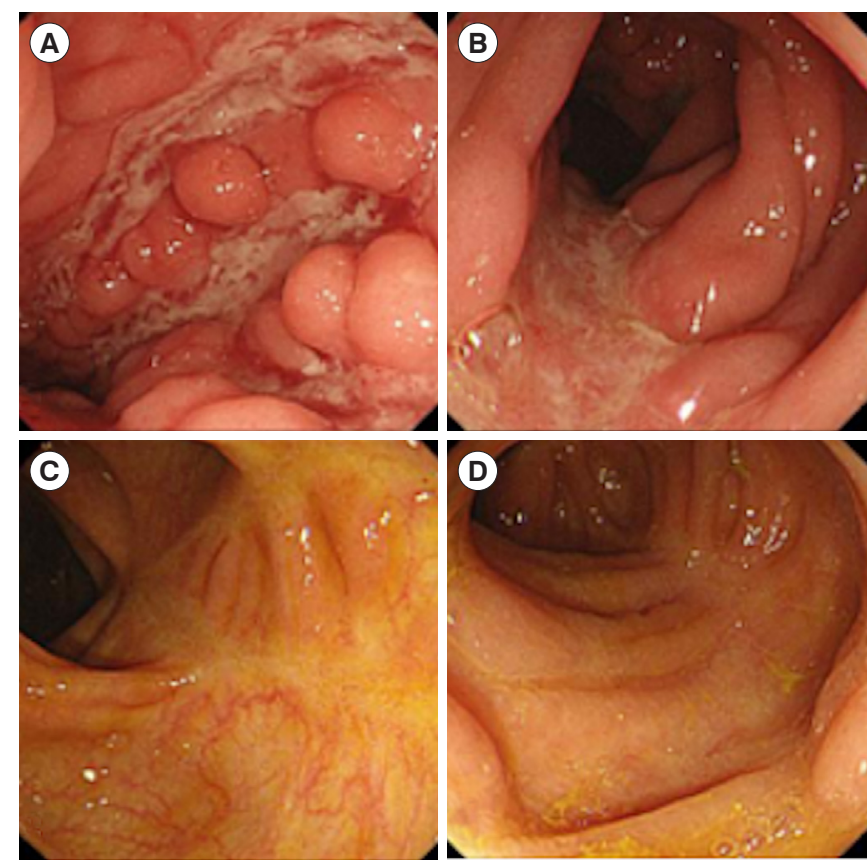

Fig. 1. Endoscopic images of the large intestine. Before hematopoietic stem cell transplantation (HSCT), multiple longitudinal ulcers in the transverse colon (A) and descending colon (B) were observed. After HSCT, these ulcers were healing in the transverse colon (C) and descending colon (D).
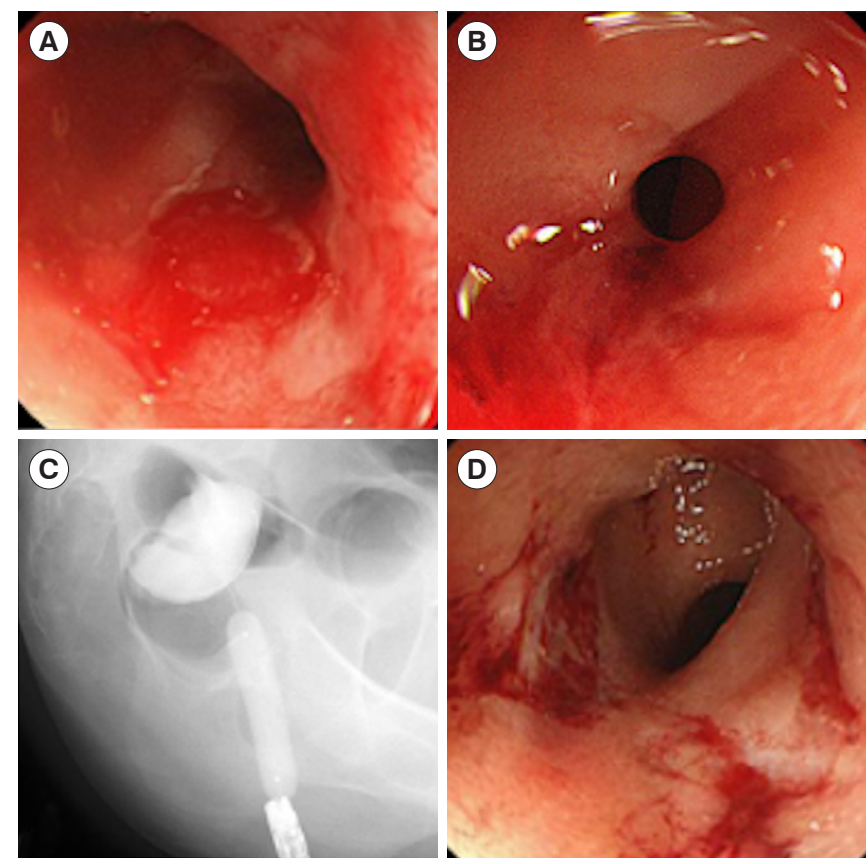

Fig. 2. Endoscopic and fluoroscopic images of the rectum. Before hematopoietic stem cell transplantation (HSCT), the rectum was mildly stenosed with active ulcers (A). After HSCT, active ulcers were healing; however, the stricture of the rectum progressed (B). We performed endoscopic balloon dilations for the rectum up to $12 \mathrm{~mm}(\mathrm{C}, \mathrm{D})$.
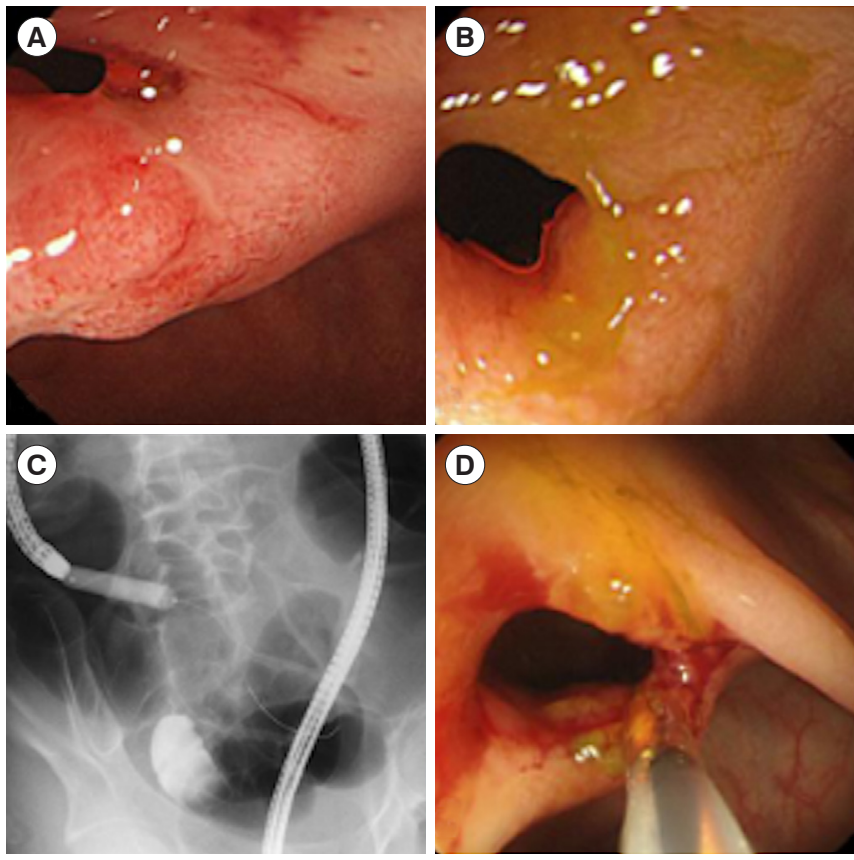

Fig. 3. Endoscopic and fluoroscopic images of the ileocecal valve (ICV). Before hematopoietic stem cell transplantation (HSCT), the ICV was severely stenosed with an active ulcer (A). After HSCT, the active ulcer was healing, and the stricture of the ICV remained severe (B). We performed endoscopic balloon dilations for the ICV up to $10 \mathrm{~mm}(C, D)$. 

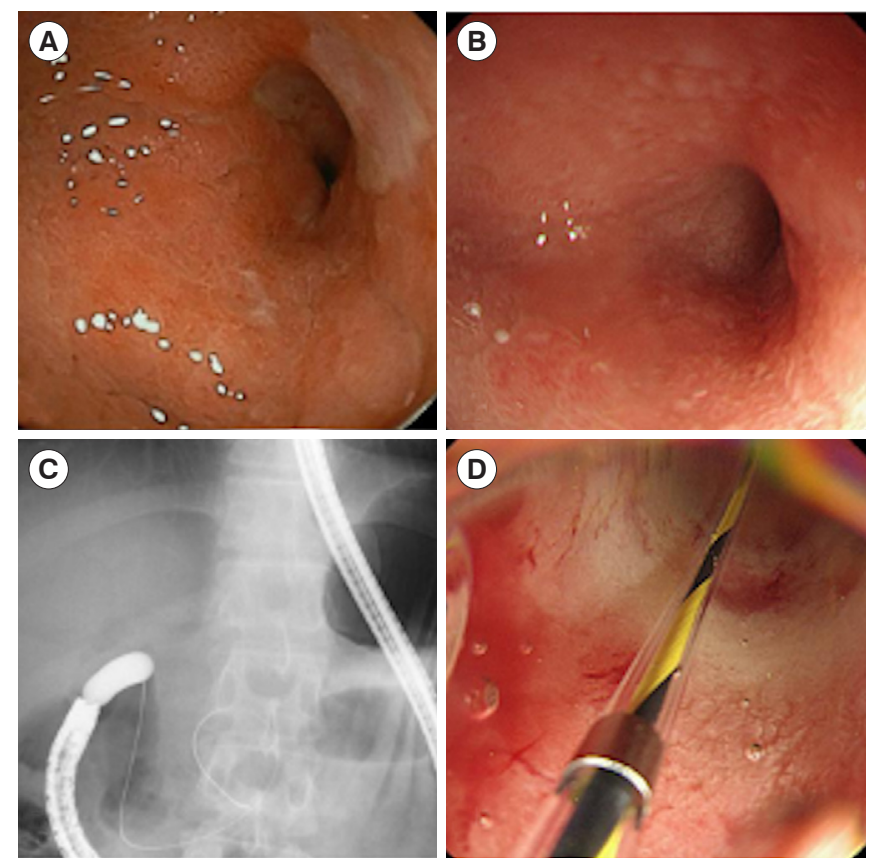

Fig. 4. Endoscopic and fluoroscopic images of the duodenum. Before hematopoietic stem cell transplantation (HSCT), the duodenum bulb was mildly stenosed with an active ulcer (A). After HSCT, the active ulcer was healing; however, the stricture of the duodenum progressed (B). We performed endoscopic balloon dilations for the rectum up to $10 \mathrm{~mm}(\mathrm{C}, \mathrm{D})$.

the nasal endoscope could not be advanced through these strictures. Since the active ulcers were healing, we decided to perform EBDs. We first dilated the stricture of the rectum using a through-the-scope balloon up to $12 \mathrm{~mm}$ (Fig. 2C and D), and the scope could be advanced. The multiple ulcers in the large intestine were healing (Fig. 1C and D), and the stricture of the ICV remained severe (Fig. 3B). Next, we dilated the stricture of the ICV up to $10 \mathrm{~mm}$ (Fig. 3C and D), and the scope could be advanced. Finally, we performed EBD for the stricture of the duodenum up to $10 \mathrm{~mm}$ (Fig. 4C and D). Throughout the patient's clinical course, no EBD complications occurred. In contrast studies, we did not find any other strictures in the small intestine (Fig. 5). He started eating after the EBDs, but abdominal symptoms did not relapse without any dietary restrictions. We have been following him for 4 months and the GI patency is maintained without any symptoms.

\section{DISCUSSION}

XIAP deficiency is mostly a pediatric, drug-resistant disease, and its symptoms are generally severe. The only curative treatment for XIAP deficiency is HSCT, and a previous study re-
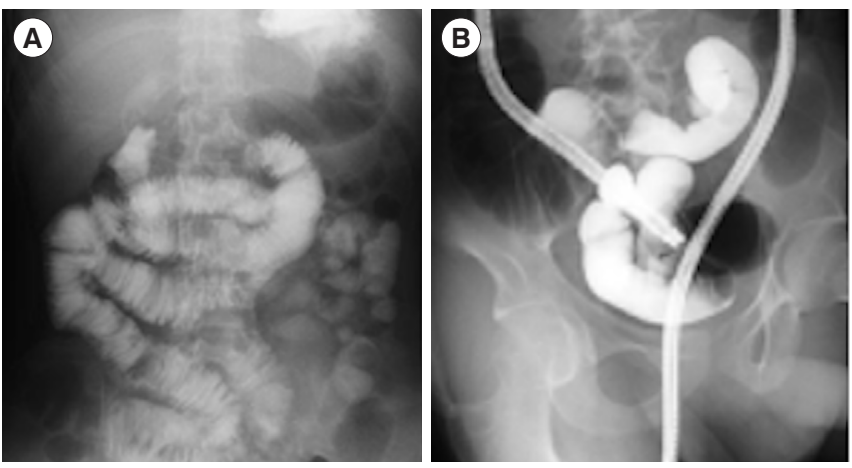

Fig. 5. Fluoroscopic images of the small intestine $(A, B)$. In contrast studies, no other stricture in the small intestine was observed.

vealed the efficacy of HSCT for GI lesions. ${ }^{8}$ In the present case, the patient had been treated with various treatments for $\mathrm{CD}$, but no drugs were remarkably effective for his GI lesions. However, mucosal healing was achieved after HSCT, as previously reported.

Little has been reported about HSCT being associated with a risk of proceeding GI strictures in patients with XIAP deficiency. In cases of CD, it was reported that rapid mucosal healing by anti-tumor necrosis factor- $\alpha$ antibodies may result in excess scar tissue formation and strictures. ${ }^{9}$ GI lesions in XIAP deficiency are similar to those in $\mathrm{CD}^{2}$ Thus, in the present case, it was possible for the fibrotic strictures to progress during the process of subsiding inflammation, as in CD.

There is no standard procedure for treating strictures in XIAP deficiency; therefore, we referred to the strategy used for treating strictures in patients with CD because of the similarity between XIAP deficiency and CD. Previous studies reported that performing EBD for intestinal strictures in patients with $\mathrm{CD}$ is safe and effective to avoid surgery. ${ }^{6}$ There are some criteria regarding EBDs for CD: (1) a short stricture $(<4 \mathrm{~cm})$ with minimal inflammation, (2) lack of malignancy or high-grade dysplasia, (3) lack of a fistula orifice close to the stricture or an abscess in the stricture area, and (4) straight strictures in line with the bowel lumen. ${ }^{10}$ Previous studies reported that main complications of EBD for CD are acute bleeding and perforations, and a larger balloon size may be associated with a higher complication rate. ${ }^{11}$ In the present case, the strictures met the criteria of EBD for CD; therefore, we chose this procedure as treatment. Our patient's strictures were very severe, so we dilated the strictures with a small balloon size (10-12 mm) to minimize the risk of complications. Consequently, no complications of EBD occurred, and clinical remission was achieved without any dietary restrictions or surgery. 
Little is known about the effects of EBD for strictures of upper GI tract in patients with CD; however, in a meta-analysis, Bettenworth et al. ${ }^{12}$ suggested that EBD for CD-associated strictures of the upper GI tract to be a valuable alternative to surgery, with a high rate of short-term technical and clinical success, moderate long-term efficacy, and an acceptable rate of complications. Like CD patients, our case suggests that EBD could be an effective and safe procedure for strictures of upper GI tract in patients with XIAP deficiency.

In conclusion, EBD could be an effective and safe procedure for intestinal strictures including upper GI tract after HSCT in patients with XIAP deficiency. XIAP deficiency patients with intestinal strictures after HSCT could avoid surgery, if EBD is properly performed.

\section{ADDITIONAL INFORMATION}

\section{Funding Source}

The authors received no financial support for the research, authorship, and/or publication of this article.

\section{Conflict of Interest}

No potential conflict of interest relevant to this article was reported.

\section{Author Contribution}

Conceptualization, data curation, formal analysis, methodology: Otagiri S. Project administration: Otagiri S, Katsurada T, Sakamoto N. Resources: Otagiri S, Katsurada T, Sakurai K, Sugita J. Supervision: Katsurada T, Sakamoto N. Visualization: Otagiri S, Sakurai K, Sugita J. Writing - original draft: Otagiri S. Writing review \& editing: all authors. Approval of final manuscript: all authors.

\section{Others}

We thank the staff of the Hokkaido University Hospital for their invaluable help with data collection. This work was supported by the Hokkaido University Hospital.

\section{ORCID}

Otagiri S

Katsurada T

Sakurai K

Sugita J
Sakamoto N

https://orcid.org/0000-0003-0061-059X

\section{REFERENCES}

1. Latour S, Aguilar C. XIAP deficiency syndrome in humans. Semin Cell Dev Biol 2015;39:115-123.

2. Aguilar C, Lenoir C, Lambert N, et al. Characterization of Crohn disease in X-linked inhibitor of apoptosis-deficient male patients and female symptomatic carriers. J Allergy Clin Immunol 2014;134:1131-1141.

3. Zeissig Y, Petersen BS, Milutinovic S, et al. XIAP variants in male Crohn's disease. Gut 2015;64:66-76.

4. Ono S, Okano T, Hoshino A, et al. Hematopoietic stem cell transplantation for XIAP deficiency in Japan. J Clin Immunol 2017;37:85-91.

5. Sunseri WM, Kugathasan S, Keljo DJ, et al. IBD LIVE case series: case 3: very early-onset inflammatory bowel disease: when genetic testing proves beneficial. Inflamm Bowel Dis 2015;21: 2958-2968.

6. Atreja A, Aggarwal A, Dwivedi S, et al. Safety and efficacy of endoscopic dilation for primary and anastomotic Crohn's disease strictures. J Crohns Colitis 2014;8:392-400.

7. Otagiri S, Sugiura R, Katsurada T, et al. Gastrointestinal: endoscopic balloon dilations for an intestinal stricture in a patient with X-linked inhibitor of apoptosis deficiency. J Gastroenterol Hepatol 2019;34:1895.

8. Tsuma Y, Imamura T, Ichise E, et al. Successful treatment of idiopathic colitis related to XIAP deficiency with allo-HSCT using reduced-intensity conditioning. Pediatr Transplant 2015;19:E25-E28.

9. Chang CW, Wong JM, Tung CC, Shih IL, Wang HY, Wei SC. Intestinal stricture in Crohn's disease. Intest Res 2015;13:19-26.

10. Klag T, Wehkamp J, Goetz M. Endoscopic balloon dilation for Crohn's disease-associated strictures. Clin Endosc 2017;50: 429-436.

11. Gustavsson A, Magnuson A, Blomberg B, Andersson M, Halfvarson J, Tysk C. Endoscopic dilation is an efficacious and safe treatment of intestinal strictures in Crohn's disease. Aliment Pharmacol Ther 2012;36:151-158.

12. Bettenworth D, Mücke MM, Lopez R, et al. Efficacy of endoscopic dilation of gastroduodenal Crohn's disease strictures: a systematic review and meta-analysis of individual patient data. Clin Gastroenterol Hepatol 2019;17:2514-2522. 